\title{
Design of WebGIS transportation and distribution system based on the genetic algorithm
}

\author{
Hongbo Hao ${ }^{1}$, Fengzhi Dai ${ }^{1,2}$ * \\ ${ }^{1}$ Tianjin University of Science and Technology, China; \\ ${ }^{2}$ Tianjin Tianke Intelligent and Manufacture Technology CO., LTD, China \\ E-mail: *daifz@tust.edu.cn \\ www.tust.edu.cn
}

\begin{abstract}
With the concept of "smart logistics" put forward, the transformation of the logistics industry facing information technology is facing great challenges. At present, China's logistics industry is facing problems such as extensive resource allocation, low management level and high cost. Aiming at the transportation problem in logistics industry, this paper designs a solution method of balanced/unbalanced transportation problem based on the Monte Carlo similarity and genetic algorithm. Two coding methods, Prufer number and matrix are adopted. On this basis, dynamic mutation rate and random mutation strategy are designed, and Monte Carlo similarity receiving method is introduced. Finally, from the perspective of system requirements, the WebGIS transportation and distribution system based on genetic algorithm is designed and developed.
\end{abstract}

Keywords: smart logistics, balanced/unbalanced, Prufer, WebGIS

\section{Introduction}

Modern logistics industry is based on information technology and transportation technology. Logistics industry is composed of goods packaging, goods handling, goods allocation, goods management and goods storage and transportation. These elements are developed around transportation.

Therefore, the study of transportation is very important and it has a strong practical significance. The transportation problem is mainly to solve the problem of allocation between producing area and selling place, and its mathematical model belongs to the category of linear programming.

The traditional algorithm is not suitable for high-dimensional transportation problems. Therefore, the intelligent algorithms such as ant colony algorithm and neural network are usually used to solve the high-dimensional transportation problems.
In this paper, a genetic algorithm based on Monte Carlo similarity is proposed for transportation problem, and Monte Carlo similarity operator is constructed to avoid the algorithm falling into local optimal solution. The research and development system has a certain practical significance for the promotion and development of intelligent logistics ${ }^{1}$.

This paper describes some basic theories involved in the algorithm, such as genetic algorithm, Monte Carlo, similarity theory and the related technical framework of WebGIS transportation and distribution system. It also includes the mathematical model and transformation relationship of the balanced/unbalanced transportation problem. An intelligent optimization algorithm based on the combination of Monte Carlo similarity and genetic algorithm is designed. The Monte Carlo similarity receiving method is introduced, and the conclusion that the genetic algorithm based on Monte Carlo and adopting matrix coding is better ${ }^{2}$. 
With the continuous enhancement of desktop processor performance, such as the WebGIS framework represented by ArcGIS API for JavaScript launched by Esri, the pressure on back-end services has been greatly reduced.

\section{Overall scheme design}

This paper uses the basic theory of genetic algorithm, Monte Carlo thought and common similarity algorithm. Then it introduces the key technology of system design and development. The front end uses bootstrap, Vue and ArcGIS API for JavaScript framework. The back end is based on the springboot framework, combined with spring, spring MVC and mybatis framework. MySQL is used as the system storage database.

\subsection{Front end architecture}

This paper uses bootstrap that is very popular nowadays. Vue.js.Bootstrap is an open source CSS framework developed by twitter engineers Jacob Thornton and mark Otto to improve code reusability. Bootstrap is a flexible, elegant, easy to use and extensible front-end framework.Vue. It is a progressive framework for building the front-end interface. Different from other frameworks, it can be applied layer by layer from bottom to top and developed based on MVVM mode Vue.JS Framework ${ }^{3}$. MVVM pattern is Vue.JS, one of the biggest features of the pattern architecture that is shown in Fig.1.

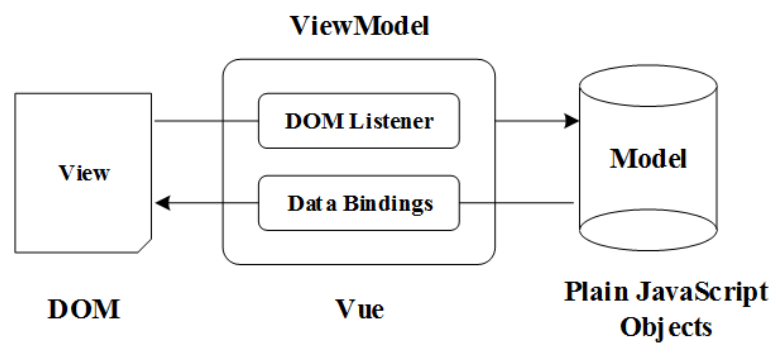

Fig.1. Vue.JS MVVM mode architecture diagram

\subsection{Back end architecture}

The Springboot solves the characteristics of various configuration, low development efficiency and complex deployment of back-end development. The Maven dependency is simplified by using start POM, and the configuration is completed by annotation. The single instance servlet improves the running efficiency. It is adopted, combined with spring, spring MVC and mybatis framework to complete the development. Therefore, spring takes IOC and AOP design patterns as the core to construct a bean container to store singleton objects. The architecture of spring is shown in Fig. 2.

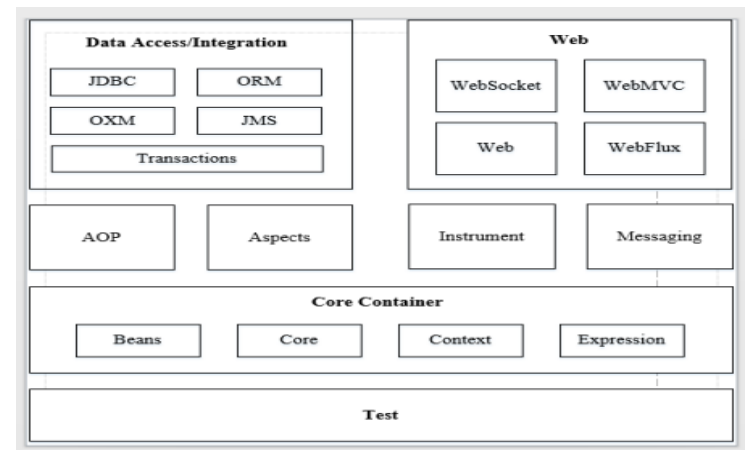

Fig.2. Spring architecture diagram

\section{Design and solution of transportation model}

\subsection{Balanced transportation model}

The equilibrium transportation problem is described as follows: suppose a product has $\mathrm{m}$ producing areas $\mathrm{W}_{1}$, $\mathrm{W}_{2} \ldots \mathrm{Wm}$ and $\mathrm{N}$ outlets $\mathrm{Q}_{1}, \mathrm{Q}_{2} \ldots \mathrm{Qn}$. Among the output of producing area is $\mathrm{W}_{\mathrm{i}}$, and the sales volume is $\mathrm{Qj}$. The cost of transporting a unit of goods from $W_{i}$ to $Q_{j}$ with a certain route $s$ in a certain period of time is $C_{i j}(t, s)$, which is represented by $\mathrm{C}_{\mathrm{ij}}$ below. Schematic diagram of origin and sale of place ${ }^{4}$ is shown in Fig. 3 .

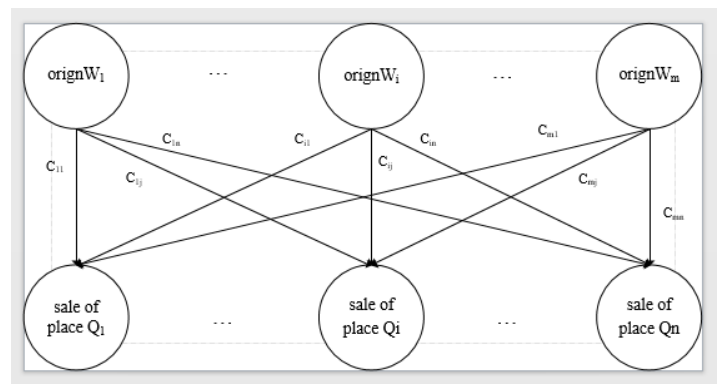

Fig.3. Schematic diagram of origin and sale of place

\subsection{Fitness function design}

The fitness function of genetic algorithm is used as an index to evaluate the quality of individuals in a population. The larger the fitness value is, the better the individual is. The fitness function is shown below.

$$
f(X)=e^{-\lambda\left[\operatorname{tr}\left(\boldsymbol{C}^{T} \boldsymbol{X}\right)-\omega\right]}
$$

\subsection{Solving transportation problems}

The algorithm process steps are as follows: 
- $\quad$ Set the operation parameters;

- Initial population;

- The fitness of each chromosome was calculated;

- The chromosomes were sorted according to the fitness;

- The first $10 \%$ individuals with high fitness were reserved;

- Monte Carlo similarity method is used to receive the difference solution between the mutated individual and the mutated parent ${ }^{5}$.

\subsection{Algorithm results and analysis}

The performance of GA algorithm based on PRüfer number coding and matrix coding are compared. It can be seen that the convergence speed and optimal value of TGA and algorithms are not as good as the improved ITGA and IMGA algorithms without using strategies.

In the process of crossover and mutation of IMGA algorithm, the generated offspring meets the constraint conditions, while the ITGA algorithm is easy to generate infeasible solutions in the crossover process.

The experimental results show that the final convergence result of IMGA algorithm is better than that of ITGA algorithm. In Fig.4, the horizontal axis is the number of iterations and the vertical axis is the average of the optimal value of each iteration in every 30 experiments. The average value of the optiomal value of each algorithm's iteration is shown in the Fig.4.

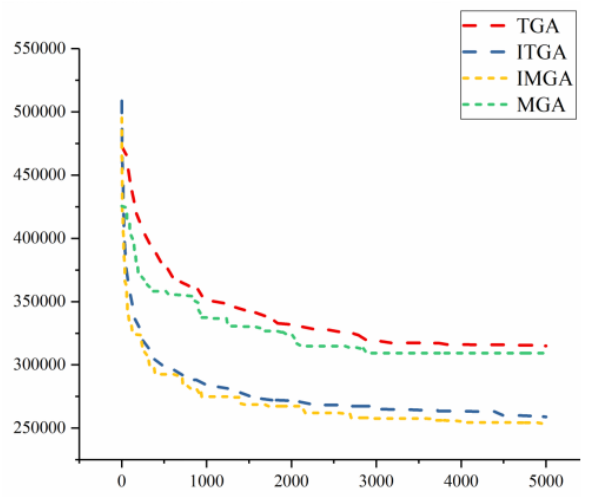

Fig.4. Average of the optimal value of each algorithm's iteration

\section{Design of WebGIS distribution system}

\subsection{System Requirements Analysis}

The WebGIS transportation and distribution system abandons the previous purely digital representation. It combines the GIS system to introduce spatial relationships into the transportation and distribution system, and selects production and sales locations in an intuitive and optional way. The system is mainly based on the task creation module. It is supplemented by task list module, OD cost module, distribution plan module, task analysis module and personal center module.

\subsection{System technical route}

The WebGIS transportation and distribution system adopts the $\mathrm{B} / \mathrm{S}$ architecture model, which reduces the burden of the client on the server and the cost of system maintenance and upgrades. The system server level uses the GIS server in ArcGIS Enterprise and the Tomcat-based Web server. The system uses the SSM framework, which is divided into a presentation layer, a business processing layer and a data persistence layer ${ }^{6}$.

\subsection{System database design}

The design of the system is mainly based on the task creation module. The table structure and relationship are designed in a task-driven way, including system user table, task list, expense table, expense detail table, distribution table, distribution details table, and algorithm analysis table, sales surface, algorithm iteration record table, etc.

\subsection{System function module design}

According to the actual system requirements, the WebGIS transportation and distribution system is equipped with various module functions of WebGIS, which are mainly divided into 6 modules: task list module, task creation module, OD cost module, distribution plan module, task analysis module and personal center module.

\subsection{System function module design}

The distribution plan module plays the role of query of calculation results in the WebGIS transportation and distribution system. This module undertakes the task creation module and the OD expense module, obtains the preprocessing data necessary for the algorithm. It calls the intelligent algorithm according to the task. The distribution plan module visually allows users to view the distribution of production and sales. With WebGIS 
technology, the production and sale area are marked in the form of a map. The number and shapes are combined to achieve the purpose of abstract digital visualization.

\section{Conclusion}

This article aims at the completeness and ease of use. The specific operation process of the WebGIS transportation and distribution system is shown in Fig5.

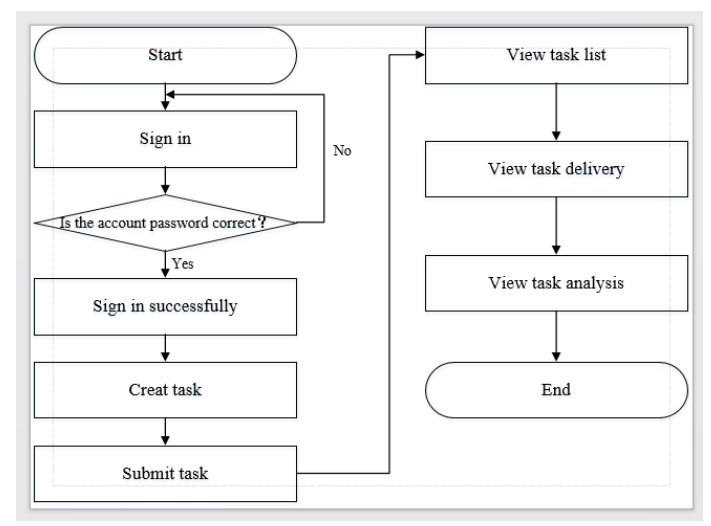

Fig.5. WebGIS distribution system flow chart

This paper takes the transportation and distribution system of a logistics company as the background. It combines the Monte Carlo similarity GA transportation problem algorithm based on matrix coding on the basis of considering the actual transportation problem requirements. The front-end framework uses Bootstrap, Vue.js and ArcGIS API for JavaScript ${ }^{7}$. The back-end framework adopts SSM and other Web system development technologies, and with the help of ArcGIS platform.

The WebGIS system that meets transportation and distribution is finally designed and realized. The system uses Intellij idea as the integrated development environment. The core algorithms and back-end services are built in Java language. The front-end development framework uses ArcGIS API for JavaScript, Bootstrap and Vue.js, etc., and data storage and management uses MySQL and ArcGIS DataStore ${ }^{8}$.

\section{Acknowledgements}

The research is partly supported by the Project of Tianjin Enterprise Science and Technology Commissioner to Tianjin Tianke Intelligent and Manufacture Technology Co., Ltd (19JCTPJC53700). It is also supported by the
Industry-University Cooperation and Education Project (201802286009) from Ministry of Education, China.

\section{References}

1. Elena Rudskaia, Rudskaia Elena, Eremenko Igor. Conceptual framework for solving problems of commercial transportation of goods in the city logistics system. IOP Conference Series: Materials Science and Engineering, 2020, 918(1): pp. 012038-.

2. Liu Lang. No-core Monte Carlo shell model calculations with unitary correlation operator method and similarity renormalization group. Chinese Physics C, 2015, 39(5).

3. Matevž Pustišek, Andrej Kos. Approaches to Front-End IoT Application Development for the Ethereum Blockchain. Procedia Computer Science, 2018, 129: pp. 410-419.

4. Meijie Ji. Study on Location Selection and Optimization of Logistics Center based on Particle Swarm Optimization. International Core Journal of Engineering, 2020, 6(9).

5. Qi Li, Rui Miao, Jie Zhang, et al. An Intelligent Recommendation Method for Service Personalized Customization. IFAC-PapersOnLine, 2019, 52(13): pp. 1543-1548.

6. YONGGANG ZUO, WEIPING ZHU. Aviation Logistics Information System Based on SSM Framework. 2017 2nd International Conference on Computer Engineering, Information Science and Internet Technology, 2017, pp. 533-538.

7. A. Taivalsaari, T. Mikkonen, D. Ingalls and K. Palacz. Web Browser as an Application Platform, 2008 34th Euromicro Conference Software Engineering and Advanced Applications, Parma, 2008, pp. 293-302.

8. Wiart Jaspar, Wong Elaine. Walsh functions, scrambled-nets, and negative covariance: Applying symbolic computation to quasi-Monte Carlo integration. Mathematics and Computers in Simulation, 2021, 182: pp. 277-295. 